\title{
Exact Tests for Correlation and for the Slope in Simple Linear Regressions without Making Assumptions
}

\author{
Karl H. Schlag
}

June 25, 2008

\begin{abstract}
We present an exact test for whether two random variables that have known bounds on their support are negatively correlated. The alternative hypothesis is that they are not negatively correlated. No assumptions are made on the underlying distributions. We show by example that the Spearman rank correlation test as the competing exact test of correlation in nonparametric settings rests on an additional assumption on the data generating process without which it is not valid as a test for correlation.

We then show how to test for the significance of the slope in a linear regression analysis that invovles a single independent variable and where outcomes of the dependent variable belong to a known bounded set.

Keywords: Correlation test, exact hypothesis testing, distribution-free, nonparametric, simple linear regression.
\end{abstract}

JEL classification codes: C12, C14, C01

\section{Introduction}

We present a test for identifying whether two variables are negatively correlated based on a finite sample of matched pairs without imposing additional assumptions. Results are proven for the given finite sample. So this is exact inference, there is no use of asymptotic theory. The method applies whenever all outcomes of each variable belong to some known bounded set (such as a closed interval). This is a condition on the data, 
not an assumption imposed by the modeler. The alternative hypothesis is formulated as the complement of the null hypothesis, thus there will be no indifference zone.

The difficulty with testing for correlation is that one cannot build on tests for independence as being uncorrelated is weaker than being independent. Permutation tests are commonly used as exact tests for independence. A special representative, the Spearman (1904) rank correlation test, is the standard test for correlation in nonparametric settings. However this test for correlation, being a permutation test, relies on a hidden assumption. It namely concludes that the data is positively correlated whenever there is sufficient evidence that the two distributions are not independent. Thus it rules out by assumption that the data is not uncorrelated but dependent. We illustrate this feature in an example and do not make such assumptions when deriving the validity of our test.

Independence and uncorrelatedness coincide when there are only two possible outcomes as is the case for Bernoulli distributions. For Bernoulli distributions there are exact tests for correlation. For instance, Tocher (1950) shows that a randomized version of Fisher's exact test, designed originally for comparing means, is in fact UMPU for testing negative covariance. Note that this test belongs to the class of permutation tests. Our test uses the equivalence between independence and uncorrelatedness in the case of binary outcomes. We first transform the problem into one of comparing two Bernoulli distributions and then apply the solution of Tocher (1950). This transformation is stochastic, creating a randomized test. An additional step is then needed to eliminate the randomness and thus to create a nonrandomized exact test.

The random transformation of the general data into binary values utilizes the given bounded sets that contain all possible outcomes. Whether or not bounds can be assigned to the possible outcomes of the variables depends on the application at hand. The larger are these bounds the weaker is the inference. We hasten to point out that nontrivial tests of correlation do not exist without such bounds without making further assumptions, extending an argument of Bahadur and Savage (1956) made for testing the mean of a single sample. To complete the picture, note that permutation tests as tests of independence do not require such bounds.

We then show how our test for correlation can be used to investigate the significance of the slope in a simple linear regression (so there is only one independent variable). We consider the classic setting of a conditional test in which it is as if outcomes of the independent variable are known before sampling. The dependent variable has unknown distribution, the statistician only knows a bounded set that contains any possible outcome. Data is generated by drawing the dependent outcomes inde- 
pendently conditional on the value of the independent variable which makes errors pairwise independent. The only assumption by the modeler on the data generating process is the linear structure. We make no restrictions on the distribution of the errors such as homoscedasticity. So we search for a test whose validity is robust to heteroscedasticity of unknown form within the limits imposed by the known bounds on the possible outcomes of the dependent variable.

To use our test for correlation for this objective we first have to show that our test is a valid conditional test for correlation, that is that it has level $\alpha$ conditional on the observed values of one of the two random variables. Thereafter the application to the simple regression is then straightforward as the covariance of the dependent and the independent variable is proportional to the coefficient of the slope.

Once again known bounds on the outcomes, here on those of the dependent variable, allow for the existence of a nontrivial test. For a discussion of lack of inference possibilities without assumptions, and hence without such bounds, in the context of regressions or econometrics more generally see Dufour (2003).

More related literature is discussed in the main section. Note that other exact tests known in the literature used to investigate correlation or slopes in a simple linear regression rely on unverifiable distributional assumptions.

\section{Test of Covariance}

Consider two random variables $Z_{1}$ and $Z_{2}$ where $Z_{i} \in\left[a_{i}, b_{i}\right]$ for some a priori known $a_{i}, b_{i} \in \mathbb{R}$ with $a_{i}<b_{i}, i=1,2$. We wish to test $H_{0}: \operatorname{Cov}\left(Z_{1}, Z_{2}\right) \leq 0$ against $H_{1}: \operatorname{Cov}\left(Z_{1}, Z_{2}\right)>0$ based on a sample of $n$ observations $\left(z_{1 i}, z_{2 i}\right)$ for $i=1, \ldots, n$ independently drawn from $Z=\left(Z_{1}, Z_{2}\right)$. Later we also consider conditional tests where inference is conditional on the observed values of $Z_{2}$.

\subsection{Importance of the Bound}

Assume that the set of outcomes of $Z_{1}$ is either unbounded or that it is bounded but where the bounds are unknown. Then there is no nontrivial test for testing the null hypothesis that $\operatorname{Cov}\left(Z_{1}, Z_{2}\right) \leq 0$. To see this consider the following example where we present the conditional probabilities of $\left.Z_{1}\right|_{Z_{2}=z_{2}}$ : 


$\begin{array}{cccc}z_{2} \backslash Z_{1} & 0 & 1 & v \\ 0 & 1-1 / m & 0 & 1 / m \\ 1 & 0 & 1 & 0\end{array}$

with $m, v>1$ but where the values of $m$ and $v$ are not known to the investigator. Note that $\operatorname{Cov}\left(Z_{1}, Z_{2}\right) \leq 0$ if and only if $v \leq m$. Yet if $m$ is sufficiently large then the event $\left.Y\right|_{X=0}=v$ occurs with arbitrary low probability and hence with arbitrarily high probability the statistician will not observe outcome $v$ and hence will not able to learn whether or not $v=m$. Consequently, any test of $\operatorname{Cov}\left(Z_{1}, Z_{2}\right) \leq 0$ rejects the null hypothesis with probability at most $\alpha$ whenever $y_{i} \neq v$ for all $i$. This means that the type II error of any level $\alpha$ test is at least $1-\alpha$ as long as there are no limits on how large $v$ can be.

Note that this example is a simple extension of the point made by Bahadur and Savage (1956), that the mean of a single sample cannot be tested if there are no restrictions on the possible distributions.

\section{$2.2 \quad$ Permutation tests}

Permutation tests are often used to uncover correlation. The most prominent representative is the Spearman (1904) rank correlation test. Permutation tests can be used to test the null hypothesis that two underlying distributions are independent. To complete this to a test for correlation one then for instance can consider as alternative hypothesis that the correlation of these two distributions is strictly positive. So one rules out by assumption that it cannot be that the two distributions are dependent but uncorrelated, as is the case in our above example when $v=m$. Note that this particular assumption can hardly be deduced from objective characteristics of the data. We do not wish to consider inference that is based on assumptions and not on the data and its objective characteristics. Hence we consider as alternative hypothesis the complement of the null hypothesis. In this sense the Spearman rank correlation test is not a valid test to uncover correlation. To complete the formal argument we provide a counter example. Assume $\alpha=0.05$. Consider the performance of the Spearman rank correlation test when facing data generated as in the above example and when $\#\left\{z_{2 i}=1\right\}=n / 2$ and $n$ even. ${ }^{1}$ Then this test recommends to reject the null hypothesis for $\alpha=0.05$ when $n \geq 8$ whenever $v$ is not observed. Clearly this does not contradict its validity as a test of independence as $Z_{1}$ and $Z_{2}$ are not independent in

\footnotetext{
${ }^{1} \# A$ denotes the cardinality of the finite set $A$.
} 
this example. However, repeating our point above, it also rejects the null hypothesis with probability $(1-1 / m)^{n / 2}$ when $v=m$ and hence when $\operatorname{Cov}\left(Z_{1}, Z_{2}\right)=0$. Thus its type I error for testing $\operatorname{Cov}\left(Z_{1}, Z_{2}\right) \leq 0$ conditional on $\left(z_{2 i}\right)_{i}$ is above 0.05 when $m$ is sufficiently large given $n$, for instance when $m \geq 4$ and $8 \leq n \leq 20$. Its invalidity as an unconditional test follows similarly provided $n$ is not too small.

\subsection{An Exact Unconditional Test}

We present an exact unconditional test. First assume that $Z_{1}$ and $Z_{2}$ are Bernoulli distributed, so $Z_{1}, Z_{2} \in\{0,1\}$. Our hypotheses are then equivalent to testing $H_{0}$ : $E\left(Z_{1} \mid Z_{2}=1\right) \leq E\left(Z_{1} \mid Z_{2}=0\right)$ against $H_{1}: E\left(Z_{1} \mid Z_{2}=1\right)>E\left(Z_{1} \mid Z_{2}=0\right)$ based on an independent sample that consists of $\#\left\{i: z_{2 i}=k\right\}$ independent draws of $\left.Z_{1}\right|_{Z_{2}=k}$ for $k=0,1$. To see this, note that

$$
\begin{aligned}
\operatorname{Cov}\left(Z_{1}, Z_{2}\right) & =\operatorname{Pr}\left(Z_{1}=Z_{2}=1\right)-\operatorname{Pr}\left(Z_{1}=1\right) \operatorname{Pr}\left(Z_{2}=1\right) \\
& =\left[\operatorname{Pr}\left(Z_{1}=1 \mid Z_{2}=1\right)-\operatorname{Pr}\left(Z_{1}=1 \mid Z_{2}=0\right)\right] \operatorname{Var} Z_{2} .
\end{aligned}
$$

So if $\operatorname{Var} Z_{2}>0$ then the equivalence is immediate. If $\operatorname{Var} Z_{2}=0$ then there are not observations of both $\left.Z_{1}\right|_{Z_{2}=0}$ and $\left.Z_{1}\right|_{Z_{2}=1}$ and hence any exact test will recommend no rejection which again shows the equivalence.

So we can use a test for comparing the means $E\left(Z_{1} \mid Z_{2}=1\right)$ and $E\left(Z_{1} \mid Z_{2}=0\right)$ to construct a one-sided test for whether covariance is negative. One such test would be the randomized version of the Fisher exact test due to Tocher (1950). Note that we have simply repeated what Tocher (1950) already pointed out, namely that the randomized version of Fisher exact test is a UMPU test for our hypotheses involving covariance.

We now use the above to construct an exact test for the more general case where the two random variables $Z_{1}$ and $Z_{2}$ are bounded. Assume that $Z_{j} \in\left[a_{j}, b_{j}\right]$ for $a_{j}<b_{j}$ and $j=1,2$. Consider the two Bernoulli random variables $Z_{1}^{\prime \prime}$ and $Z_{2}^{\prime \prime}$ such that

$$
\begin{aligned}
\operatorname{Pr}\left(Z_{1}^{\prime \prime}=Z_{2}^{\prime \prime}=1\right) & =\frac{E Z_{1}-a_{1}}{b_{1}-a_{1}} * \frac{E Z_{2}-a_{2}}{b_{2}-a_{2}} \\
\operatorname{Pr}\left(Z_{1}^{\prime \prime}=1, Z_{2}^{\prime \prime}=0\right) & =\frac{E Z_{1}-a_{1}}{b_{1}-a_{1}} * \frac{b_{2}-E Z_{2}}{b_{2}-a_{2}} \\
\operatorname{Pr}\left(Z_{1}^{\prime \prime}=0, Z_{2}^{\prime \prime}=1\right) & =\frac{b_{1}-E Z_{1}}{b_{1}-a_{1}} * \frac{E Z_{2}-a_{2}}{b_{2}-a_{2}} .
\end{aligned}
$$

These Bernoulli random variables emerge under the following algorithm. First renormalize $Z_{j}$ with a linear transformation such that outcomes are in $[0,1]$. Then trans- 
form any outcome $z_{j}$ realized of $Z_{j}$ with probability $z_{j}$ into into 1 and with probability $1-z_{j}$ into 0 where the random transformation of $z_{j}$ occurs independently of the transformation of $z_{3-j}, j=1,2$.

Note that $E Z_{j}^{\prime \prime}=\left(E Z_{j}-a_{i}\right) /\left(b_{i}-a_{i}\right)$. More importantly,

$$
E\left(Z_{1}^{\prime \prime} Z_{2}^{\prime \prime}\right)=E\left(Z_{1} Z_{2}\right) /\left[\left(b_{1}-a_{1}\right)\left(b_{2}-a_{2}\right)\right]
$$

and hence

$$
\operatorname{Cov}\left(Z_{1}^{\prime \prime}, Z_{2}^{\prime \prime}\right)=\operatorname{Cov}\left(Z_{1}, Z_{2}\right) /\left[\left(b_{1}-a_{1}\right)\left(b_{2}-a_{2}\right)\right] .
$$

Thus, the randomized version of Fisher exact test can be used for testing $H_{0}$ : $\operatorname{Cov}\left(Z_{1}, Z_{2}\right) \leq 0$ against $H_{1}: \operatorname{Cov}\left(Z_{1}, Z_{2}\right)>0$. Replace any observation $z_{j i}$ of $Z_{j}$ in the sample with 1 with probability $\left(z_{j i}-a_{j}\right) /\left(b_{j}-a_{j}\right)$ and with 0 with probability $\left(b_{j}-z_{j i}\right) /\left(b_{j}-a_{j}\right)$. Then apply the UMPU test to the transformed sample. This generates a randomized test that has size $\alpha$. Moreover it is unbiased. In fact, since the underlying test is UMPU for the Bernoulli case this means that this randomized test for the nonparametric setting minimizes the type II error among all unbiased tests whenever the alternative hypothesis only depends on the expectations and on the covariance. Formally, this is when $H_{1}:\left\{Z:\left(E Z_{1}, E Z_{2}\right) \in W_{1}, \operatorname{Cov}\left(Z_{1}, Z_{2}\right) \in W_{2}\right\}$ for some $W_{1} \subseteq\left[a_{1}, b_{1}\right] \times\left[a_{2}, b_{2}\right]$ and $W_{2} \subseteq \mathbb{R}^{+} \backslash\{0\}$.

Now we sketch how to construct a nonrandomized test (which will no longer be unbiased). The procedure is as follows. Fix some threshold $\theta \in(0,1)$ that may not depend on the data gathered on $Y$ but only on the independent variables. Calculate the expected probability of rejection when using the UMPU test with level $\theta \alpha$. Reject the null hypothesis if this expected probability is above $\theta$. This nonrandomized test has level $\alpha$. Moreover its type II error can be bounded above. The reasoning follows the analysis of Schlag (2007) who presents a test for comparing means. Schlag (2007) also present several methods for selecting $\theta$, to prevent choice of $\theta$ based on the data we set the default equal to $\theta=0.2$ (see also discussion below).

\subsection{An Exact Conditional Test}

We now show that the above randomized and nonrandomized unconditional tests are in fact also valid exact conditional tests, so when inference in terms of the covariance is conditional on the observed values of $Z_{2}$. This conditional approach is valuable when the values of $Z_{2}$ are known before the sampling takes place such as when they are determined by the investigator. It will be enough to consider the randomized test as the construction of the nonrandomized test from the randomized test is a general 
valid procedure that does not depend on the fact that we are here testing for negative correlation.

Assume that $\left(z_{2 i}\right)_{i}$ is known (and nonrandom) before running the test but that $\left.Z_{1}\right|_{Z_{2}=z_{2}}$ remains unknown. The sample $\left(z_{1 i}\right)_{i}$ is generated by drawing $z_{1 i}$ independently drawn from $\left.Z_{1}\right|_{Z_{2}=z_{2 i}}$. Let $Z_{2}^{\prime}$ be the random variable where $\operatorname{Pr}\left(Z_{2}^{\prime}=z_{2}^{\prime}\right)=$ $\#\left\{i: z_{2 i}=z_{2}^{\prime}\right\} / n$. Then

$$
\operatorname{Cov}\left(Z_{1}, Z_{2}^{\prime}\right)=\frac{1}{n} \sum_{i=1}^{n} E\left(Z_{1} \mid Z_{2}=z_{2 i}\right) z_{2 i}-\left(\frac{1}{n} \sum_{i=1}^{n} E\left(Z_{1} \mid Z_{2}=z_{2 i}\right)\right)\left(\frac{1}{n} \sum_{i=1}^{n} z_{2 i}\right) .
$$

We wish to test $H_{0}: \operatorname{Cov}\left(Z_{1}, Z_{2}^{\prime}\right) \leq 0$ against $H_{1}: \operatorname{Cov}\left(Z_{1}, Z_{2}^{\prime}\right)>0$. Of course, such a test will also be valid for $H_{0}: \operatorname{Cov}\left(Z_{1}, Z_{2}\right) \leq 0$ against $H_{1}: \operatorname{Cov}\left(Z_{1}, Z_{2}\right)>0$ where $Z_{2}$ is the random variable from which $\left(z_{2 i}\right)_{i}$ have been drawn, not necessarily independently of each other.

In contrast to the above setting, $\left(z_{2 i}\right)_{i}$ is no longer a random sample from $Z_{2}^{\prime}$. We claim that the above unconditional randomized test is an exact test for this conditional inference. Analogously to above we find that $\operatorname{Cov}\left(Z_{1}^{\prime \prime}, Z_{2}^{\prime \prime}\right)=\operatorname{Cov}\left(Z_{1}, Z_{2}^{\prime}\right) /\left[\left(b_{1}-a_{1}\right)\left(b_{2}-a_{2}\right)\right]$ where $Z_{2}^{\prime \prime}$ is derived from $Z_{2}^{\prime}$ in the same way $Z_{2}^{\prime \prime}$ was derived from $Z_{2}$ above. Given the construction of our test we only have to consider the properties of the test when $Z_{1}$ is Bernoulli distributed, $\left(z_{2 i}\right)_{i} \in\{0,1\}^{n}$ and when we are testing $H_{0}$ : $\operatorname{Pr}\left(Z_{1}=1 \mid Z_{2}^{\prime}=1\right) \leq \operatorname{Pr}\left(Z_{1}=1 \mid Z_{2}^{\prime}=0\right)$ against $H_{1}: \operatorname{Pr}\left(Z_{1}=1 \mid Z_{2}^{\prime}=1\right)>\operatorname{Pr}\left(Z_{1}=1 \mid Z_{2}^{\prime}=0\right)$. The validity of using our unconditional test as conditional test is possibly lost as we no longer have iid observations of $\left(Z_{1}, Z_{2}^{\prime}\right)$. In fact, generally $\operatorname{Pr}\left(Z_{1}=1 \mid Z_{2}=z_{2 i}\right)$ will depend will depend on $i$. Consider however the special case where $\operatorname{Cov}\left(Z_{1}, Z_{2}^{\prime}\right)=0$. Then either $\operatorname{Var} Z_{2}^{\prime}=0$ in which case the null hypothesis is not rejected or $\operatorname{Var} Z_{2}^{\prime}>0$ and $\operatorname{Pr}\left(Z_{1}=1 \mid Z_{2}^{\prime}=1\right)=\operatorname{Pr}\left(Z_{1}=1 \mid Z_{2}^{\prime}=0\right)$. In the latter case this means that $\operatorname{Pr}\left(Z_{1}=1 \mid Z_{2}=z_{2 i}\right)$ is independent if $i$. So if $\operatorname{Cov}\left(Z_{1}, Z_{2}^{\prime}\right)=0$ then our unconditional test has type I error $\alpha$ regardless of how $\left(z_{2 i}\right)_{i}$ was created. Now consider the case where $\operatorname{Cov}\left(Z_{1}, Z_{2}^{\prime}\right)<0$. This means that $\operatorname{Pr}\left(Z_{1}=1 \mid Z_{2}^{\prime}=1\right)<\operatorname{Pr}\left(Z_{1}=1 \mid Z_{2}^{\prime}=0\right)$. Note that our above test is based on the UMPU test of Tocher (1950) which has the property that the null hypothesis is more likely to be rejected if $\operatorname{Pr}\left(Z_{1}=1 \mid Z_{2}^{\prime}=1\right)$ is increased. This means that the type I error of our test is attained for the case where $\operatorname{Pr}\left(Z_{1}=1 \mid Z_{2}^{\prime}=1\right)=\operatorname{Pr}\left(Z_{1}=1 \mid Z_{2}^{\prime}=0\right)$ but here we argued above that the type $\mathrm{I}$ error is equal to $\alpha$. This means that the unconditional test has size $\alpha$ conditional on the realized values of $Z_{2}$ and hence it is also a valid exact conditional test. Moreover our arguments show that it again is best (in terms of minimizing type II error) among all unbiased tests provided the alternative hypothesis can be formulated in terms of 
$E Z_{j}$ and $\operatorname{Cov}\left(Z_{1}, Z_{2}\right)$ only.

\subsection{Discussion}

\subsubsection{Comparing Means}

We presented our exact test for correlation as an extension of a test for the binary valued case to distributions with support contained known bounded sets. Note however that, after the random transformation into a problem involving Bernoulli distributions, it is really only about comparing two success probabilities $\operatorname{Pr}\left(Z_{1}^{\prime \prime}=1 \mid Z_{2}^{\prime \prime}=1\right)$ and $\operatorname{Pr}\left(Z_{1}^{\prime \prime}=1 \mid Z_{2}^{\prime \prime}=0\right)$. There are other tests besides the UMPU test for this objective. For instance, Schlag (2007) provides evidence that the Z test (see Suissa and Shuster, 1984) performs better when the sample is almost balanced while the UMPU test does better when the sample is very unbalanced. Balancedness refers to there being approximately equally many realizations of $Z_{2}^{\prime \prime}=1$ and of $Z_{2}^{\prime \prime}=0$. Thus a more sophisticated test of correlation would involve choosing different tests for comparing means conditional on the realization of $Z_{2}^{\prime \prime}$.

Given this connection to tests for comparing success probabilities one can in fact choose the same values of the threshold $\theta$ as used when comparing success probabilities using the UMPU test. This is why we suggest $\theta=0.2$ as default as this was the typical parameter discovered when comparing means (Schlag, 2007). A more sophisticated test would use a threshold $\theta$ that depends on the sample size $n$ and possibly on the sampled values of $Z_{2}$ (such as through its empirical variance). Different methods for selecting $\theta$ are presented in Schlag (2007). Note that for proof of the exactness of this test $\theta$ may not depend on the transformed outcomes $Z_{2}^{\prime \prime}$.

A connection to tests for comparing means also emerges when we show that known bounds on outcomes are necessary to ensure nontrivial tests of correlation. Here we reiterate arguments by Bahadur and Savage (1956) originally formulated for testing the mean of a single sample but easily extended to comparing two means.

\subsubsection{Variations}

Analogously one can construct a conditional test for $H_{0}: \operatorname{Cov}\left(Z_{1}, Z_{2}\right) \geq 0$ against $H_{1}: \operatorname{Cov}\left(Z_{1}, Z_{2}\right)<0$. Together with the above one can then create equi-tailed conditional tests of zero covariance, so where $H_{0}: \operatorname{Cov}\left(Z_{1}, Z_{2}\right)=0$ against $H_{1}$ : $\operatorname{Cov}\left(Z_{1}, Z_{2}\right) \neq 0$.

Similarly one may be interested in tests that refer to correlation coefficient $\rho\left(Z_{1}, Z_{2}\right)=$ 
$\operatorname{Cov}\left(Z_{1}, Z_{2}\right) / \sqrt{\operatorname{Var} Z_{1} \cdot \operatorname{Var} Z_{2}}$ with the convention that $\rho=0$ when $\operatorname{Var} Z_{1} \cdot \operatorname{Var} Z_{2}=$ 0 . Note that $\operatorname{Var} Z_{1} \cdot \operatorname{Var} Z_{2}>0$ then $\rho\left(Z_{1}, Z_{2}\right) \leq 0$ if and only if $\operatorname{Cov}\left(Z_{1}, Z_{2}\right) \leq 0$. Moreover, $\operatorname{Var} Z_{1} \cdot \operatorname{Var} Z_{2}=0$ implies $\operatorname{Cov}\left(Z_{1}, Z_{2}\right)=0$. Hence our above conditional test for covariance can be used as conditional test for $H_{0}: \rho\left(Z_{1}, Z_{2}\right) \leq 0$ against $H_{1}: \rho\left(Z_{1}, Z_{2}\right)>0$.

\section{Simple Linear Regression}

We now use our above test to construct an exact test of the significance of the slope in a simple linear regression.

Consider the following standard simple linear regression model that consists of a single independent variable $X$ and a dependent variable $Y$. We depart from the classic framework by considering a dependent variable that generates outcomes that belong to a known compact set. Let $\left(x_{i}\right)_{i=1}^{n}$ be the outcomes of $X$ that are known to the investigator, so we will be making inference conditional on the values of $X$. Let $Y$ be a random variable that generates outcomes in $[a, b]$ for known values $a$ and $b$ with $a<b$ where $E\left(Y \mid X=x_{i}\right)=\beta_{0}+\beta_{1} x_{i}$ where $\beta_{0}, \beta_{1} \in \mathbb{R}$ are unknown. Inference is based on a sample $\left(y_{i}\right)_{i=1}^{n}$ of outcomes realized by $Y$ conditional on $x_{i}$ where specifically $y_{i}$ is drawn from $\left.Y\right|_{X=x_{i}}$ independently of the other draws for each $i=1, . ., n$. We wish to test $H_{0}: \beta_{1} \leq 0$ against $H_{1}: \beta_{1}>0$.

\subsection{Exact Approaches}

We first review some other approaches to this problem to then conclude that there is no existing exact approach that does not rely on additional assumptions on the data when the dependent variable has bounded support.

\subsubsection{Classic Approach}

In the classic exact approach to linear regression one assumes that errors are normally distributed. This is not applicable (in its exact formulation) when data has the property that all possible outcomes of the dependent variable belong to some compact set. This is because errors cannot be normally distributed as the normal distribution has unbounded support. In addition, the classic approach is also only exact when all errors have the same variance (homoscedasticity). This assumption is hard to verify from the data and typically does not follow directly from properties of the underlying data generating process. Note that we do not assume homoscedasticity. 
The typical regression analysis does not rely on the finite sample exact approach but instead assumes that the sample is sufficiently large, relying on the fact that the $\mathrm{p}$ values of the exact approach are approximately correct provided the sample is sufficiently large. The problem with using this methodology (also referred to as asymptotic theory) here is that there is no upper bound on how large the sample has to be. This is because the upper bound depends on the underlying data generating process which itself is unknown. The approximation is not uniform. In fact it turns out that this approach of relying on the sample being sufficiently large can be arbitrarily imprecise. For instance, consider the case where there are only two possible outcomes of the independent variable of a simple regression. Then the standard tests of significance are based on the two sample t test. For this test it is known (see arguments of Lehmann and Loh, 1990) that without homoscedasticity there is no upper bound on the sample size that makes the $\mathrm{p}$ values approximately correct independently of the underlying data generating process. In fact, the size of the test is known to be 1 .

One way out of this dilemma, while retaining mathematically correct statements, is to add additional assumptions on the data generating process such as imposing bounds on higher moments. One could then explicitly derive the precision of the $\mathrm{p}$ values derived under normality as functions of the imposed bounds, however this is not done in practice.

\subsubsection{Other Exact Approaches in the Literature}

There are some alternative exact approaches, however these involve imposing additional assumptions. Daniels (1954) assumes that the distribution of errors does not depend on dependent variable and has median 1/2. Sievers (1978) considers a simple linear regression where the distribution of errors does not depend on independent variable.

\subsection{An Exact test for Significance of the Slope}

We wish to test $H_{0}: \beta_{1} \leq 0$ against $H_{1}: \beta_{1}>0$. The claim is that this can be done exact at level $\alpha$ by evaluating our above test for negative correlation to the data $\left(x_{i}, y_{i}\right)_{i=1}^{n}$.

To verify this claim consider the random variables $X^{\prime}$ and $Y^{\prime}$ such that $\operatorname{Pr}\left(X^{\prime}=\xi\right)=$ $\#\left\{i: x_{i}=\xi\right\} / n$ and $\operatorname{Pr}\left(Y^{\prime}=\eta\right)=\#\left\{i: y_{i}=\eta\right\} / n$. These variables arise when choosing equally likely one of the $n$ observations $i$ and then setting $X^{\prime}=x_{i}$ and 
$Y^{\prime}=y_{i}$. Let $a_{1}=\min _{i}\left\{x_{i}\right\}$ and $b_{1}=\max _{i}\left\{x_{i}\right\}$. Then $X^{\prime} \in\left[a_{1}, b_{1}\right]$ and $Y^{\prime} \in[a, b]$. The sample can be interpreted as an independent sample of $\left.Y^{\prime}\right|_{X^{\prime}=x_{i}}$ conditional on $\left(x_{i}\right)_{i}$ that have been realized by $X^{\prime}$. Moreover, $\operatorname{Cov}\left(X^{\prime}, Y^{\prime}\right)=\beta_{1} \operatorname{Var}\left(X^{\prime}\right)$. We have thus established the necessary conditions to apply our above test to investigate whether $\operatorname{Cov}\left(X^{\prime}, Y^{\prime}\right) \leq 0$. Note that this will also be a test of whether $\beta_{1} \leq 0$ as $\left\{\left(X^{\prime}, Y^{\prime}\right): \beta_{1} \leq 0\right\} \subset\left\{\left(X^{\prime}, Y^{\prime}\right): \operatorname{Cov}\left(X^{\prime}, Y^{\prime}\right)\right\}$.

\section{References}

[1] Bahadur, R. R. and Savage, L. J. (1956), "The Nonexistence of Certain Statistical Procedures in Nonparametric Problems," Annals of Mathematical. Statistics, 27, $1115-1122$.

[2] Daniels, H. E. (1954), "A Distribution-Free Test for Regression Parameters," Annals of Mathematical Statistics, 25(3), 499-513.

[3] Dufour, J.-M. (2003), "Identification, Weak Instruments, and Statistical Inference in Econometrics," Canadian Journal of Economics, 36(4), 767-808.

[4] Lehmann, E. L. and Loh, W.-Y. (1990), "Pointwise verses Uniform Robustness in some Large-Sample Tests and Confidence Intervals," Scandinavian Journal of Statistics, 17, 177-187.

[5] Schlag, K. H. (2007), Testing Equality of Two Means without Assumptions - Solving the Nonparametric Behrens-Fisher Problem, European University Institute, Unpublished Manuscript (Available from http://www.eui.eu/Personal/Schlag/papers/2meaninfer.pdf).

[6] Sievers, G. L. (1978), "Weighted Rank Statistics for Simple Linear Regression," Journal of the American Statistical Association, 73(363), 628-631.

[7] Spearman, C. (1904), "The Proof and Measurement of Association Between Two Things," American Journal of Psychology, 15, 72-101.

[8] Suissa, S. and Shuster, J. J. (1984) "Are Uniformly Most Powerful Unbiased Tests Really Best?" American Statistician, 38, 204-206.

[9] Tocher, K. D. (1950), "Extension of the Neyman-Pearson Theory of Tests to Discontinuous Variates," Biometrika, 37, 130-144. 